\title{
PENSAMIENTO CRÍTICO EN ESTUDIANTES DEL GRADO DE EDUCACIÓN SOCIAL ${ }^{1}$
}

\section{Critical thinking in Social Education Grade students}

\author{
Francisco José García Moro \\ Universidad de Huelva \\ Correo-e: fjose.garcia@dpsi.uhu.es \\ Walter Federico Gadea Aiello \\ Universidad de Huelva \\ Correo-e: walter.gadea@ddi.uhu.es \\ Vicente de Jesús Fernández Mora \\ Universidad de Huelva \\ Correo-e: vicente.fernandez@ddi.uhu.es \\ Recibido: 18 de junio de 2020 \\ Envío a informantes: 30 de junio de 2020 \\ Aceptación definitiva: 14 de septiembre de 2020
}

RESUMEN: El presente artículo se centra en una investigación cualitativa sobre las creencias acerca del pensamiento crítico que poseen 72 estudiantes de tercer curso del Grado de Educación Social de la Universidad de Huelva (España). Los resultados, en consonancia con otras investigaciones, muestran que el alumnado tiene un conocimiento poco detallado aunque certero de lo que significa dicha competencia, ofreciendo explicaciones generales que van en acuerdo con las ideas que tienen acerca de la Universidad, la Sociedad y la finalidad de la Educación Social. La buena disposición que muestra la mayoría del alumnado investigado choca con escasos hábitos para el pensamiento crítico, achacándolo a una formación reproductiva, a la influencia perniciosa de determinados poderes sociales y a un no-saber-hacer-y-actuar de los futuros educadores sociales.

Palabras Clave: pensamiento crítico; educación social; creencias; formación superior.

Artículo fruto de la ayuda recibida por la Universidad de Huelva al amparo de la convocatoria de Proyectos de innovación docente 20I8-2019 para el desarrollo del proyecto AULA DE DEBATE: LA CONSTRUCCIÓN DEL PENSAMIENTO CRÍTICO, LA EXPRESIÓN CORPORAL Y LA ORATORIA. 
AвSTRACT: This article focuses on qualitative research on the beliefs about critical thinking held by 72 third year students of the Social Education Degree of the University of Huelva (Spain). The results show that the students have a little detailed but accurate knowledge of what this competence means, offering general explanations that are in line with the ideas they have about the University, Society and the purpose of Social Education. The willingness shown by the majority of the students researched clashes with scarce habits for critical thinking, blaming it on reproductive training, the pernicious influence of certain social powers and the non-know-how-to-act of future social educators.

KEY WORDs: critical thinking; social education; beliefs; higher education.

\section{Introducción}

$\mathrm{E}$ L PENSAMIEnto CRítico constituye una de las competencias fundamentales que por definición debe caracterizar el hacer del educador social; y más aún el buen hacer y buen ser del educador social. Es una habilidad que se aprende y que, por tanto, requiere de tiempo y dedicación para que forme parte consustancial y habitual del repertorio comportamental del que asume la función de proponer posibilidades a la sociedad. Consideramos que el educador social debe tener la competencia y habilidad de saber escrutar lo que se le presenta, valorando su idoneidad para lo que necesita la sociedad más que lo que dice que necesita.

Saber decidir lo que es importante de lo que es nimio en función de las propias necesidades y de las exigencias de una sociedad que está agazapada tantas veces en apariencias resulta una competencia que no se puede considerar precisamente baladí, por el contrario, es una competencia que debería desarrollar toda persona y más aquella que tiene el cometido profesional de dedicarse «al otro» para el cambio positivo. Por otra parte, en un mundo tan sobreinformado como el nuestro (Sánchez, Ferrán, Baiges y Suárez, 20I9), donde la «infobesidad» (Wolton, 20Io) domina la esfera informativa de nuestra sociedad de consumo, la única forma de acceder al potencial comunicativo que ofrece tanta información es utilizando la capacidad crítica como estrategia de búsqueda de lo adecuado, necesario y correcto.

A la hora de aproximarse al concepto de pensamiento crítico, vemos qué se ha hecho y hace desde diferentes disciplinas, buscando cada una de ellas su desarrollo y su aplicación (Altuve, 20I0; Valencia y Puente, 20I8; Vélez, 2013). Esto ha complicado sobremanera su conceptualización y evaluación. Diferentes disciplinas como la filosofía, las ciencias sociales, la educación, la psicología, las didácticas de las ciencias, entre otras, han reflexionado y reflexionan sobre el pensamiento crítico, su finalidad específica y, como efecto colateral, agudizando la falta de consenso epistemológico y conceptual de lo que es. Aun a pesar de esta dispersión de caracterizaciones debido a su naturaleza multicompleja (Valenzuela y Nieto, 2008; Vélez, 2013), algunas de las definiciones arrojan luz sobre lo que se entiende por pensamiento crítico, teniendo en cuenta que nos podemos centrar en un contínuum que va desde la reflexión teorética a una investigación-acción para y en la práctica que busca, tal y como apunta Morales (20I4), el cambio social. Así, el pensamiento crítico se ha definido como: (a) un juicio autorregulado para un propósito específico que depende de la propia persona (Facione, I990); (b) una estrategia de pensamiento, investigación y proceso (Boisvert, 
2004); (c) una forma de reflexión racional e intuitiva que nos permita comprender el mundo actual (Altuve, 20Io); (d) una capacidad que se adquiere y que permite el razonamiento y que reflexiona sobre qué decidir y qué hacer (Ennis, 20II); (e) un pensar por uno mismo de una forma activa y reflexiva (Febres, Alirio y Africano, 20I7); (f) un juicio basado en datos objetivos y subjetivos previamente interpretados y analizados (Olivares y López, 2017); (g) un modo de pensar reflexivo e intencionado alternativo al habitual en el que se activan recursos cognitivos (Valenzuela y Nieto, 2008); (h) un modo de aprender a formular y resolver preguntas y encontrar conclusiones acertadas a partir de la observación y la información (Paul y Elder, 2003); (i) una barrera contra la excesiva información que trata de convencernos de cualquier cosa (Epstein, 2006); (j) un pensamiento rico conceptualmente que está organizado de forma coherente y que busca, de una forma persistente, la exploración de lo que aparece (Lipman, 1998).

Por su parte, Sánchez, Ferrán, Baiges y Suárez-Guerrero (2019) resumen esta diversidad conceptual en las siguientes posibilidades: (a) capacidad para expresar una postura personal; (b) expresar un punto de vista, más o menos fundamentado; (c) emitir un juicio; (d) habilidad de pensamiento que es parte de un proceso cognitivo más complejo; (e) actitud reflexiva que supone conocimientos, habilidades analíticas, comprensión y procesamiento de la información.

Bezanilla, Poblete, Fernández, Arranz y Campo (2018) indican que el pensamiento crítico está orientado a la comprensión de los problemas, pero también a su resolución, evaluando alternativas para la toma de decisiones. Implica, por tanto, comprender, evaluar para resolver. Este carácter de compromiso y transformación social es el que fundamentalmente nos interesa en nuestra investigación, puesto que contextualizar el pensamiento crítico es darle a la estrategia una finalidad que, en este caso, la legitima y le da valor (García, 2019).

A estas definiciones habría que añadir aquellas con un talante más contestatario de transformación social. Toda la pedagogía crítica vivida por Paulo Freire y «su» llamada humana al cambio de una sociedad tan humanamente desigual, pero con posibilidades. También, todas las aportaciones de la teoría crítica, influida por las ideas de la Escuela de Fráncfort, destacando a personalidades como Wilfred Carr, Basil Berstein, Stephen Kemmis, Michael Apple, Peter MaClaren, Henri Giroux. También hay que destacar a personalidades como Frantz Fanon y pensadores de la talla de Matthew Lipman, Marcuse, Habermas, Dewey, entre tantos otros.

Sea como fuere, asumimos una finalidad más aplicada, que ya de por sí está reflejada en la propia idiosincrasia del pensamiento crítico, sin minusvalorar las aportaciones más teóricas puesto que, en el campo que nos ocupa como es el de la educación social, ambas son indisociables. En esta línea, nos interesa especialmente el pensamiento crítico como medio para transformar realidades que van en contra del bien de la persona en sociedad. Este principio supone competencias para interpelar críticamente lo que aparece ante la persona en sociedad y esta competencia no termina con el «darse cuenta de que algo no es cierto o bueno o adecuado», sino que dándome o dándonos cuenta de ello implementamos las estrategias necesarias para amortiguar los daños, potenciar lo positivo, utilizando los mecanismos sociales y personales necesarios (García, 2015). Estamos de acuerdo con Facione (1990), cuando dice que el pensamiento crítico no es sinónimo de buen pensamiento, pero que aun así se constituye en un elemento que permite autorrectificar. Por otra parte, en nuestro campo de acción -la acción social en general y la educación social en particular- si bien es 
importante conocer los entresijos, estructuras y componentes del pensamiento crítico, obviamente resulta más importante y fundamental utilizarlo como un medio que puede vehicular las fuerzas necesarias para propiciar el cambio social a la luz de los principios fundamentales de una sociedad de derechos y deberes.

El pensamiento crítico, al menos en la educación para el cambio social, va mucho más lejos del dominio de ciertas habilidades cognitivas, requiriendo de competencias sociales que posibiliten la solución de problemas en un contexto real y en un tiempo real (Halpern, 1998). Pero aún más, estudios como los de Sánchez, Ferrán, Baiges y Suárez (2019) muestran que, si bien hay una consciencia por parte del alumnado universitario de la importancia del pensamiento crítico, valorando la importancia de contrastar y aplicar la crítica sobre las informaciones que les llegan, los alumnos siguen teniendo importantes deficiencias para llevarla a la práctica. Parece que hay una importante falta de coherencia entre lo que se dice y lo que realmente se hace; entre las intenciones y pretensiones y las acciones. En referencia a los docentes universitarios (Bezanilla et al., 20I8), tales estudios muestran que muchos docentes reconocen la importancia del pensamiento crítico, aunque pocos lo incorporan a sus asignaturas, puesto que parece que muy pocos entienden realmente el concepto. En este sentido, Thompson (20II) reflexiona sobre la falta de claridad por parte de los docentes universitarios sobre el concepto y lo que supone, traduciéndose esta falta de claridad en el hecho de no entender realmente bien el concepto. No obstante, no cabe duda de que el pensamiento crítico forma parte normalmente de los idearios curriculares de las universidades como competencia transversal que debe mostrar el alumnado al finalizar su formación. Sin embargo, hay que tener en cuenta que hay docentes que no lo tienen claro, no tanto su idoneidad sino su posibilidad; en este sentido, el área de estudio, la madurez del alumnado en cuestión, el ideario de la universidad, el enfoque desde el que se parte y entiende el pensamiento crítico son elementos determinantes. Tal y como señalan Bezanilla et al. (20I8), es importante aclarar el concepto a trabajar puesto que no es lo mismo el pensamiento crítico que busca favorecer el análisis y organización que aquel que, teniendo en cuenta esto, busca tomar decisiones para la transformación social desde el compromiso.

En el campo de la educación social, el pensamiento crítico lo podríamos definir como la competencia de darse cuenta de que alguna situación, hecho, información, pensamiento, sentimiento, decisión, circunscrito al ámbito personal y/o sociocomunitario, no se adecua a lo que es, teniendo en cuenta que se entiende por lo que es la defensa del bien de la persona en sociedad y sus derechos y deberes fundamentales, movilizando las disposiciones personales necesarias para argumentar y proponer alternativas, favoreciendo la conciencia y consciencia social a través de sus legítimas vías (García, Chaves y Gadea, 20I9). Esta definición tiene en cuenta las posiciones críticas contra aquel pensamiento crítico que ha perdido su carácter de transformación social, valorando la importancia de las variables ideológicas, sociales y culturales por encima de un proceso cognitivo individual (Gonzalo, 2019).

\section{Método}

A la luz de lo dicho hasta ahora y teniendo en cuenta nuestra ocupación en el campo de la formación de los futuros «transformadores de realidades» (García, 20I5) en 
el ámbito sociocomunitario, quisimos hacer una investigación acerca de las creencias y opiniones de 72 estudiantes del penúltimo curso de la carrera de Grado de Educación Social de la Universidad de Huelva (España), acerca de diferentes cuestiones relacionadas directamente con el pensamiento crítico (Tabla I). La distribución de los estudiantes por sexo y edad es la que aparece en las Tablas 2 y 3 . El diseño de la investigación se basa en el estudio de casos buscando la descripción detallada en función de cuestiones de investigación.

El único criterio de inclusión para participar en el estudio fue estar matriculado en la asignatura Competencias psicológicas del profesional de la educación social, la cual se imparte en el tercer curso del grado mencionado; no contemplamos como criterio de exclusión, como en otros estudios, los malos resultados académicos puesto que, aunque como apuntan Franco, Almeida y Saiz (2014), el pensamiento crítico es un recurso fundamental que se relaciona con el rendimiento académico y el éxito en la vida cotidiana, los criterios e instrumentos de evaluación de la universidad puede ser que en ocasiones bloqueen dicho desarrollo, tal y como exponen Moreno y Velázquez (2017), Vélez (2013), aunque realmente lo quieran potenciar.

En concreto, se creó un cuestionario ad hoc utilizando la plataforma formulario de Google, redactando diferentes cuestiones, tanto en escala tipo Likert como abiertas, para analizar el grado de acuerdo que el alumnado tenía con aspectos relacionados con el pensamiento crítico en la sociedad, en la universidad, en la carrera y en ellos mismos. Como se recoge en la Tabla ı, las cuestiones están pensadas para indagar los conocimientos que tienen los estudiantes investigados sobre el concepto de pensamiento crítico, sobre cuáles son sus características. Hay que tener presente que, en los planes de estudio de la titulación, no aparece como materia de estudio específicamente hablando dicha competencia, aunque sí aparece como competencia que debe adquirir el futuro profesional de la educación social, por lo que el conocimiento -a priori- del alumnado analizado es, en el caso de que lo tenga, por acciones propias de la autoformación fundamentalmente.

Además de analizar las ideas generales sobre qué entienden por pensamiento crítico, también nos interesaba saber en qué lugar dejan a la universidad en esta competencia y, sobre todo, a la titulación de educación social.

TABLA I. Cuestiones planteadas para el análisis

\section{Cuestiones del formulario}

a) Para mí es importante un pensamiento crítico

b) Mi grado de conocimiento de lo que es el pensamiento crítico es

c) Considero que la universidad ya favorece de una forma implícita el pensamiento crítico

d) La sociedad actual favorece posiciones críticas

e) Para las personas con las que habitualmente me desenvuelvo fuera de la universidad, es importante el pensamiento crítico

f) Para las personas con las que habitualmente me desenvuelvo en la universidad, es importante el pensamiento crítico 
TABla i. Cuestiones planteadas para el análisis (cont.)

\section{Cuestiones del formulario}

g) Las materias cursadas en la universidad favorecen, en su mayoría, el pensamiento crítico

h) A lo largo de mis estudios universitarios he tenido asignaturas que han tenido como objetivo explícito desarrollar el pensamiento crítico

i) La sociedad actual exige personas con pensamiento crítico que sepan discriminar lo fundamental de lo accesorio

j) Considero que sé discriminar lo esencial de lo accesorio en mi vida

k) En mi titulación es fundamental el pensamiento crítico

1) En mi futuro profesional es fundamental el uso del pensamiento crítico

m) Considero que tengo capacidad para argumentar más allá de las opiniones en los temas relevantes para la transformación social

n) Considero que sería muy positivo que en las enseñanzas universitarias se impartieran talleres y cursos que favorecieran el pensamiento crítico y la capacidad argumentativa

o) He realizado cursos, talleres, lecturas, etc., sobre pensamiento crítico y competencia argumentativa

Fuente: Elaboración propia.

TABla 2. Datos de la muestra-sexo

\begin{tabular}{lcccc}
\hline sexo & & & & \\
\hline \multirow{4}{*}{ Válido } & Fombrecuencia & Porcentaje & Porcentaje válido \\
& mujer & 59 & 18,1 & 18,1 \\
& Total & 72 & 81,9 & 81,9 \\
& & 13 & 100,0 & 100,0
\end{tabular}

Fuente: Elaboración propia.

Tabla 3. Datos de la muestra-edad

\begin{tabular}{lcccc}
\hline edad & & & & \\
\hline & & Frecuencia & Porcentaje & Porcentaje válido \\
\hline \multirow{2}{*}{ Válido 24} & 64 & 88,9 & 88,9 \\
& $\geq$ de 25 & 8 & 11,1 & 11,1 \\
& Total & 72 & 100,0 & 100,0
\end{tabular}

Fuente: Elaboración propia. 
PENSAMIENTO CRÍTICO EN ESTUDIANTES

DEL GRADO DE EDUCACIÓN SOCIAL

F. J. GARCÍA MORO, W. F. GADEA AIELLO Y V. DE J. FERNÁNDEZ MORA

En el análisis de la información se utilizaron, para las informaciones de frecuencias, el SPSS versión 23, así como el procedimiento de la Grounded Theory (Corbin y Strauss, 2008; Páramo, 2015), identificándose unidades y proposiciones con significación en la temática planteada, codificación de dichas unidades y proposiciones, para agruparlas en función de núcleos temáticos que permitan la construcción de categorías que posibiliten su análisis. Para el análisis cualitativo de las trascripciones de los 72 cuestionarios se utilizó el software ATLAS.ti.7

\section{Resultados y discusión}

De los 72 cuestionarios recibidos, se utilizaron y transcribieron el Ioo\% de los mismos, analizando más de 620 códigos; siendo dicho análisis la base fundamental en la que nos basamos debido a las inexistentes correlaciones significativas y diferencias significativas que se encontraron con el análisis estadístico cuantitativo a la hora de analizar relaciones bivariadas o de muestras independientes. En este sentido, no se encontraron diferencias significativas en función del sexo ni de la edad.

Centrándonos en el análisis hermenéutico de las aportaciones de los 72 participantes en el estudio contrastándolo con las aportaciones de la investigación teórica, y analizando el perfil «alumnado de cursos superiores del Grado de Educación Social», obtuvimos resultados interesantes. En este sentido, el roo\% de la muestra analizada considera fundamental e importante que el futuro educador o educadora social posea una capacidad crítica que le permita generar cambios positivos en el trasfondo social. Este valor que se le da al pensamiento crítico como estrategia de transformación social contrasta con el hecho de que el Ioo\% de los estudiantes investigados consideran que no tienen una adecuada formación en esta competencia ni teórica ni aplicada. A su vez, son especialmente críticos con la universidad en este sentido, en tanto en cuanto que el $92 \%$ de los investigados consideran que la universidad no favorece planteamientos críticos que favorezcan el cambio. Y si nos referimos a la sociedad actual, prácticamente el $99 \%$ de los estudiantes consideran que los «grandes hermanos sociales» como los mass media, la publicidad, la política, etc., tienen más bien un poder narcotizante que bloquea la capacidad crítica y, más aún, la competencia para transformar la sociedad en un lugar de oportunidades.

Un dato interesante del estudio que merece una reflexión aparte es que, si bien, como mencionamos, el $100 \%$ de los investigados consideran fundamental el pensamiento crítico, existe entre estos mismos una gran resistencia a la hora de ponerlo en práctica, hasta el punto de que más del $86 \%$ no se sienten cómodos bien porque les supone un gran esfuerzo o complicación, bien porque requiere de constancia y disciplina, etc. Del ıoo\% que inicia actividades que requieren de pensamiento crítico, solo un $15 \%$ las finaliza de forma adecuada a los planteamientos de dicha competencia. Este dato es especialmente significativo teniendo en cuenta que previamente se ha dado formación específica sobre el tema en cuestión a la totalidad del alumnado y siempre posteriormente a la recogida de los cuestionarios de análisis para así no contaminar las respuestas. En esta línea, coincidimos con las conclusiones alcanzadas por otros estudios, como los de Moreno y Velázquez (2017), en los que se constatan las dificultades que presentan los estudiantes a la hora de analizar la información, a la hora de proponer alternativas adecuadas a los diferentes interrogantes propuestos $\mathrm{y}$, 
en definitiva, a manifestar un pensamiento reproductivo y lineal, alejado de planteamientos divergentes y críticos.

Vamos a analizar los resultados obtenidos de una forma más detallada en función de las siguientes categorías de estudio: (a) cómo definen/conceptualizan el pensamiento crítico; (b) cuáles son los componentes que caracterizan a una persona con competencia crítica; (c) cómo valoran a la universidad en el desarrollo de esta competencia; (d) cómo relacionan la Educación Social con el pensamiento crítico.

\section{I. ¿Cómo definen el pensamiento crítico?}

El alumnado objeto de la presente investigación tiene una gran dificultad a la hora de conceptualizar lo que se entiende por pensamiento crítico, aun teniendo en cuenta la heterogeneidad de definiciones dependiendo de la disciplina en la que nos situemos, tal y como hemos visto en la parte introductoria. De hecho, en un primer momento del cuestionario, se tuvieron que dar por inválidas las respuestas de los estudiantes debido al escaso desarrollo de sus respuestas. Solicitando explícitamente una respuesta más elaborada y/o justificada a la cuestión de lo que, como educadores y educadoras sociales, entendían por pensamiento crítico, encontramos respuestas con una mayor fundamentación, aunque sin abandonar la generalidad, tal y como se refleja en la Tabla 4.

Del conjunto de definiciones aportadas por los investigados, no encontramos ninguna en la que se haga referencia a la intervención social, ni incluso a la intervención en general. Las definiciones que dominan son aquellas en las que se valora defender la propia opinión; la capacidad de comprender lo que se dice; la estrategia para dirimir lo importante de lo anecdótico. Obviamente, este tipo de creencias sobre lo que entiende por pensamiento crítico reflejan una forma de entender la educación social en particular y la educación en general; la finalidad social parece obviarse focalizando el protagonismo en estrategias del intelecto centradas en el uno, sin una dimensión relacional. En este sentido, consideran que se tiene pensamiento crítico si: (a) Se sabe defender las propias opiniones; sin valorar si las propias opiniones son adecuadas, ciertas u oportunas. En esta línea, resulta interesante el concepto de Teorías Implícitas para comprender el peso que tienen las propias creencias como sistema de actuar sobre y en el mundo, más que la formación o lo que se dice. (b) Se sabe identificar las ideas que alienan a la persona procedentes de la publicidad, la política, etc. No obstante, no queda claro si esa confrontación también afecta a las propias ideas. La respuesta de «sé que está mal» sin un porqué claro, justificado y descrito domina las acciones inmediatas del alumnado. (c) Se confunde la preconcepción de ideas, algo innato y natural a la persona pensante y sintiente, con el hecho de trascenderlas en el caso de que la valoración de lo que hay, en función de lo que debe ser a la luz del bien de la persona en sociedad, así lo aconseje. (d) Sea como fuere, si analizamos las definiciones más allá del contexto de la educación social que legitima nuestra investigación, se observa que las mismas no están mal encaminadas. En este sentido no están tan alejada de la siguiente definición: «Un pensar por uno mismo de una forma activa y reflexiva» (Febres, Alirio y Africano, 2017). Sin embargo, esta conceptualización y praxis de la competencia crítica se reduce a una herramienta para lo privado, para la conducta individual frente a lo que nos viene de fuera; constituyéndose en retos cognitivos y toma de decisiones circunscrita a lo próximo (Gonzalo, 2019). 

a) El pensamiento que se debe tener a la hora de querer cambiar algo [9:3].
b) La forma en que las personas analizan todo aquello que ven [4:4].
c) Poner en práctica los conocimientos adquiridos de una manera correcta [II:2].
d) Pensamiento fundamentado en algún tema, con el objetivo de expresar tu opinión de forma constructiva [14:3].
e) Tu punto de vista y los múltiples pensamientos y conocimientos que tienes acerca de un tema desde la profundidad de un tema, sabiendo y conociendo el tema desde el interior [i:I].
f) Un proceso en el que analizamos la información, que por norma general se da por ver- dadera sin pararte a analizarla [72:3]. y analizar las situaciones que se le presentan en la vida de manera constructiva [70:7]. la misma [16:2].
g) La capacidad que tiene una persona para ver más allá de lo aparente, saber reflexionar
h) La capacidad para analizar una situación y ver las características positivas y negativas de
i) El pensamiento propio que elabora una persona sobre algo mediante la búsqueda de información [18:5].
j) La capacidad de poder expresar nuestra opinión sobre cualquier tema sin tener ideas preconcebidas ni influencias externas [19:2].
k) La capacidad para expresar conocimientos e ideas de manera ordenada y lógica, anali- zando la información desde distintos puntos de vista y diferentes perspectivas [24:2].
1) Es tener un pensamiento propio que no sea influido y que vaya más allá de la percepción [25:5].
m) Capacidad de cuestionar y decidir por uno mismo [32:3].
n) Tener tus propias ideas acerca de un tema sin que te puedan influir los pensamientos de los demás [38:2].
o) Tener tus propios ideales sin dejarte guiar por algunas influencias [44:2].
p) Es un proceso que se propone analizar, entender y evaluar la manera en la que se orga- nizan los conocimientos que se pretende interpretar [48:3].
q) La capacidad para razonar las cosas que están ocurriendo, y no creernos lo que nos dicen los medios [54:4].
r) Para mí el pensamiento crítico es aquella forma de pensar cada actuación, analizar todos los sucesos que ocurren para después darle una u otra interpretación, en función del momento y situación en la que nos encontremos [57:I].
s) El pensamiento crítico es no dar por válida ninguna información sin haberla sometido anteriormente a un profundo y exhaustivo cuestionamiento que nos permita verificar por nosotros mismos dicha información, matizarla, posicionarnos al respecto, etc. [58:6].
t) Capacidad de depurar la información que recibimos sabiendo diferenciar la buena infor- mación de la mala información y la información basura [66:3].

Fuente: Elaboración propia.

\section{2. ¿Cómo caracterizan a la persona con competencia crítica?}

Ya hemos visto que la persona que es competente en pensamiento crítico se caracteriza por su capacidad argumentativa, por su capacidad de analizar lo que aparece 
para centrarse en lo que hay de cierto y/o conveniente, buscando la solución de problemas y tomando decisiones de forma eficaz para conseguir los resultados deseados (Saiz y Rivas, 2008). En este sentido, y en un primer momento, el alumnado tenía clara la imagen del pensador crítico, pero le costaba describirla; salvo algunos comentarios que confundían esta competencia con una posición contestataria irracional que para nada tiene que ver con la finalidad de dicho pensamiento en la educación social, la mayoría de los comentarios se acercaban a lo que los diferentes investigadores aportan sobre el tema. En este sentido, en la Figura i aparecen las codificaciones más representativas que salen después del análisis de los cuestionarios. Podemos observar que las características más nombradas y descritas son las que se indican a continuación: razonadora, evaluadora, curiosa, con pensamiento propio y culta. En concreto, el alumnado encuestado considera que la persona con competencia crítica sabe razonar, argumentando con conocimiento de causa sus ideas; a su vez, muestra una actitud curiosa e interesada por el mundo y las personas que lo conforman, lo que le hace salir muchas veces de su zona de confort para implicarse. Además, no se deja llevar por las modas, sino que tiene su propio pensamiento, sus ideas y principios avalados por el conocimiento, la formación y el estudio. Este pensamiento propio y cultura le permiten ser más empático con los problemas e ideas de los «otros».

Se comprueba cómo los componentes cognitivos y disposicionales están presentes, siendo el alumnado coherente, aunque de forma general, con las propuestas de los investigadores en esta línea; así, Facione (1990) ya recoge el acuerdo del panel de experto sobre pensamiento crítico en el que se identifican dos dimensiones básicas para su configuración (Tabla 5).

Figura i. Características del pensador crítico.

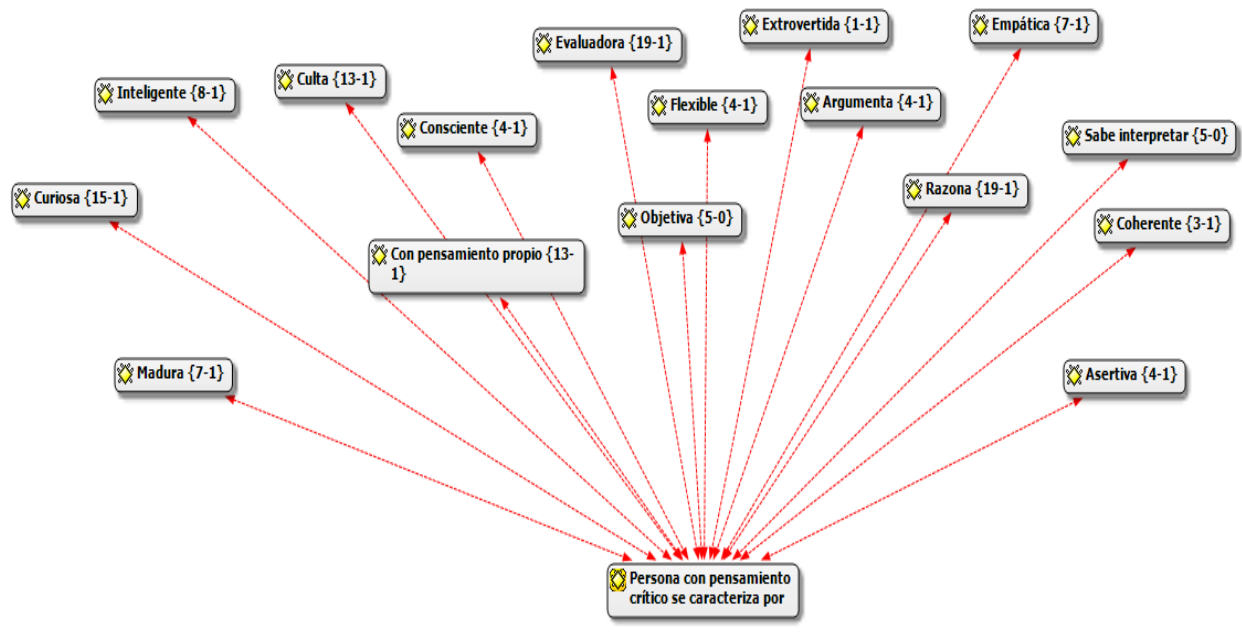

Fuente: Elaboración propia. 
Tabla 5. Dimensiones básicas del pensamiento crítico

1. Habilidades cognitivas:

a. Interpretación.

b. Análisis.

c. Evaluación.

d. Inferencia.

e. Explicación.

f. Autorregulación

2. Disposiciones o actitudes personales:
a. Inquisitiva.
b. Sistémica.
c. Juiciosa.
d. Buscadora de la verdad.
e. Analítica.
f. Mente abierta.
g. Confianza en el razonamiento.

Fuente: Facione (1990).

Por su parte, Valenzuela, Nieto y Muñoz (20I4) indican que existe un consenso en los teóricos del pensamiento crítico de que esta manera de pensar está constituida por dos componentes: las habilidades, entendidas como el dominio de procedimientos para alcanzar un razonamiento correcto, y voluntad para querer utilizar el pensamiento crítico. En este sentido, resulta interesante el hecho de que más del $86 \%$ de los encuestados informan que el esfuerzo que puede suponer el proceso crítico es un obstáculo para llevarlo a cabo.

\section{3. ¿Cómo valoran el papel que la universidad le da al pensamiento crítico?}

González y Morillo (2018) indican que el pensamiento crítico es una constante en los discursos educativos actuales; sin embargo, esta tendencia constante contrasta con la ausencia de propuestas prácticas en este sentido, produciéndose una gran distancia entre las finalidades enunciadas, los propósitos y competencias establecidos como deseables y las prácticas reales de enseñanza que pretende desarrollar el pensamiento crítico. En esta misma línea, los autores alertan de una importante paradoja con la que nos encontramos a la hora de analizar las representaciones sociales del profesorado, $y$ es que toman como punto de partida lo que dicen y no tanto lo que hacen. Queda claro que el papel del profesor universitario y de la universidad es fundamental en el hecho de favorecer el desarrollo del pensamiento crítico entre los estudiantes. Ante la opinión generalizada de la importancia del pensamiento crítico nos encontramos que no siempre se sigue una relación causal entre cómo se piensa la enseñanza y cómo se practica.

Centrándonos en los estudiantes objeto de estudio, el 92\% de los mismos muestran un desencanto con la formación que se está recibiendo en la titulación universitaria, no tanto referida a la preparación y profesionalidad de los docentes, sino a la urdimbre de la propia enseñanza. No tanto a la falta de consciencia sobre la importancia del pensamiento crítico, sino de la dificultad para llevarlo a cabo. 
Tal y como se puede observar en la Figura 2, el alumnado cree que la universidad sí que tiene en cuenta, al menos teóricamente (en sus memorias y guías docentes) el desarrollo de facto del pensamiento crítico pero le cuesta ya que «nos enseñan a tener un pensamiento supeditado a los tiempos establecidos en las programaciones y en la propia dinámica de la universidad, alejado de la sociedad» [38:6]; no obstante, aunque se tiene en cuenta en la universidad, no de una forma general o extendida a todas las titulaciones o asignaturas. En este sentido, «no en todas las titulaciones se da, bien por la propia dificultad de la titulación o porque da poco juego» [48:3], incluso en una misma titulación «algunas asignaturas son más proclives que otras para el desarrollo del pensamiento crítico, aunque esto puede ser debido a las características del profesor, o a su metodología, al alumnado en cuestión o a la posibilidad de la propia asignatura» [47:5]. Sea como fuere, hay un importante acuerdo (el $99 \%$ del alumnado encuestado) que considera que la metodología dominante en la titulación que están cursando es reproductiva, dejando como algo residual planteamientos más creativos incardinados en la problemática social y en un planteamiento de escrutador social y actor social propios del rol del educador y la educadora social. «Las clases magistrales que dominan las asignaturas de la carrera aunque se presenten con toques creativos algunas de ellas» [43:5] no parecen ayudar en la línea de fortalecer el pensamiento crítico, más aún cuando «se ciñe a los conocimientos aptos para poder aprobar, simplificando la riqueza y dificultad de lo que nos podemos encontrar en la sociedad» [46:7]. En un sistema en el que se «impone todo lo que hay que estudiar para aprobar y todo está planificado y temporalizado...» [51:2], es fácil acomodarse, incluso dándose el caso de que esta acomodación y conformismo epistemológico no venga «por la metodología utilizada, que puede favorecer el pensamiento crítico, sino por la propia costumbre de tener que estudiar para aprobar» [41:3].

Figura 2. Universidad-pensamiento crítico.

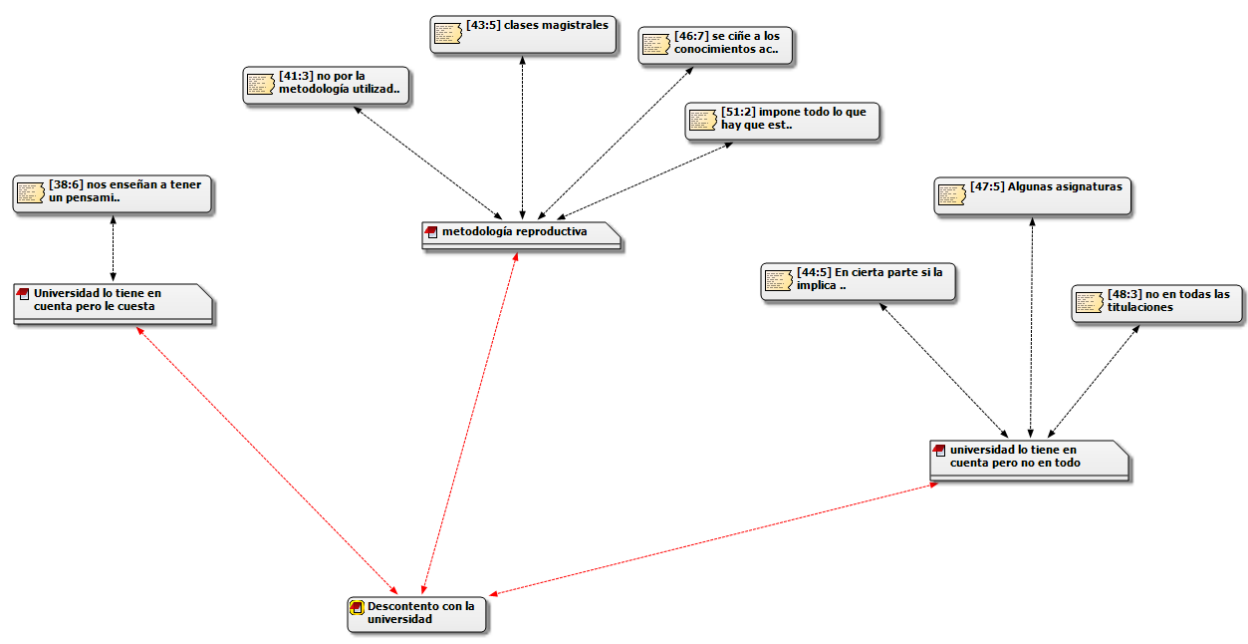

Fuente: Elaboración propia. 
Estamos de acuerdo con Moreno y Velázquez (2017) cuando afirman que la realidad actual de nuestra sociedad requiere de «ciudadanos formados con una mentalidad crítica, abierta y flexible ante los cambios. Enfrentar esos retos requiere de sistemas educativos que destaquen por la aplicación de métodos de enseñanza que conduzcan a potenciar las habilidades de pensamiento crítico y la formación integral de los estudiantes» (p. 54), con el objetivo último de contribuir a la transformación de la realidad.

\section{4. ¿¿ué papel juegan el pensamiento crítico y la educación social?}

Por último, en cuanto a la relación entre pensamiento crítico y educación social, nos encontramos que el roo\% de la muestra considera que el profesional de la educación social debe tener competencia crítica, considerándolo algo fundamental en la formación del mismo. Posteriormente, cuando se le pregunta al alumnado por qué es importante dicha competencia en dicho rol profesional, aparecen las siguientes respuestas (Figura 3). El pensamiento crítico se ve como una competencia necesaria que se debe enseñar en la formación inicial de la educación social, «Para ser educadores sociales debemos de tener un pensamiento crítico y debería ser enseñado en la carrera...» [34:7], ya que «La importancia que tiene nuestro modo de entender y actuar en la sociedad no debería dejarse al azar o a las propias creencias...» [41:4], puesto que «Trabajamos con personas con dificultades que necesitan de nuestra orientación» [47:6] y «sin un mínimo de pensamiento crítico no deberíamos trabajar en este campo» [43:7]. Hay que tener claro que el objetivo de la educación social «es enseñar a defender los derechos de los más vulnerables» [35:4], «ser capaces de enseñar al otro...» [38:19].

Figura 3. Educación social-pensamiento crítico.

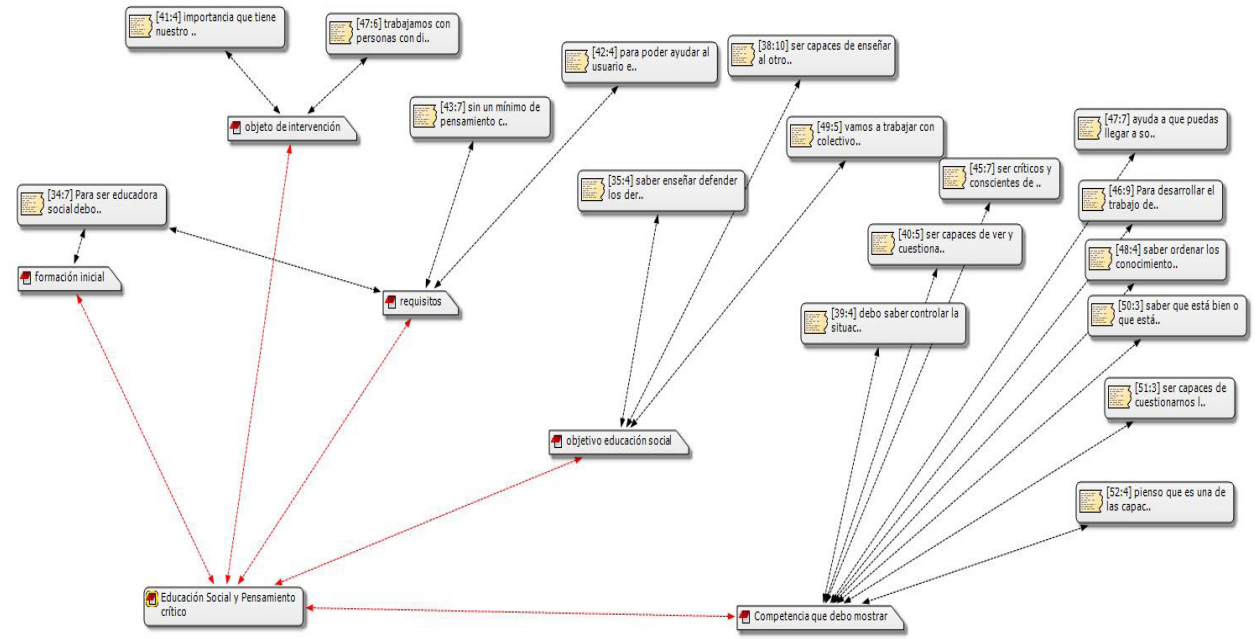

Fuente: Elaboración propia. 
Consideran que el pensamiento crítico es fundamental en la educación social porque: (a) «Debo saber controlar la situación con la que debo trabajar» [39:4]; (b) «Ser capaces de ver y cuestionar lo que nos presentan» [40:5]; (c) «Ser críticos y conscientes de lo que pasa en la sociedad» [45:7]; (d) «Ayuda a que puedas llegar a solucionar problemas» [47:7]; (e) «Para desarrollar el trabajo de forma adecuada...» [46:9]; (f) «Saber ordenar los conocimientos necesarios para poder trabajar.» [48:4]; (g) «Saber qué está bien o qué está mal.» [50:3]; (h) «Ser capaces de cuestionarnos las cosas» [51:3].

Si bien la importancia que sí le dan al pensamiento crítico queda clara, también el $95 \%$ de la muestra indican que no saben exactamente desarrollar un pensamiento crítico, quedando el $5 \%$ representado por posiciones de «no sabe/no contesta». Cuando se observa el comportamiento de la muestra en las diferentes sesiones de trabajo a lo largo de cuatro meses en dos sesiones a la semana de dos horas, se comprueba que el alumnado fundamentalmente reproduce estrategias y actitudes sin plantearse la idoneidad de la misma, incluso sin cuestionar la información que se ofrece; siendo el papel del docente fundamental a la hora de propiciar el insight necesario para propiciar el cambio. Se observa cómo la falta de habituación se convierte en una disposición limitadora en el desarrollo de competencias críticas independientemente de la importancia y/o conocimiento que se le dé y/o tenga el pensamiento crítico en el campo de la educación social.

\section{Conclusiones}

El pensamiento crítico -su conceptualización, análisis, desarrollo y aplicaciónconstituye un elemento fundamental en la formación de los futuros profesionales y más de aquellos que se van a dedicar a la educación para el desarrollo pleno social y personal como son los educadores y educadoras sociales. Esta importancia, reconocida por los que actualmente se están formando y por quienes forman, no siempre va acompañada de acciones prácticas reales; así, la intención general de lo que me parece como deseable es lo que parece dominar el quehacer de los futuros educadores sociales. El hacer las cosas que siempre se han hecho; reproducir lo que se me ha enseñado sin un cuestionamiento que lleve a la acción, al cambio, personal y social es lo que domina el quebacer de la muestra investigada. Queda claro, en este estudio, que aun siendo importante el conocimiento de lo que significa y supone la competencia crítica, incluso otorgándole un papel fundamental en la formación inicial y desempeño profesional del educador y la educadora social, no se tiene claro lo que supone y, más aún, no se observa una motivación mantenida de llevarla a cabo, fundamentalmente por el esfuerzo que implica sostenerla en el tiempo. Ante esta realidad, los docentes tenemos un papel fundamental en la formación para el cambio de los futuros profesionales; una importancia que debe trascender la que se le da realmente en la universidad para desarrollar acciones específicas, programadas y explícitas de formación en esta competencia tan fundamental para el desarrollo personal y profesional en una sociedad que requiere de competencia crítica como la actual. 
PENSAMIENTO CRÍTICO EN ESTUDIANTES

DEL GRADO DE EDUCACIÓN SOCIAL

F. J. GARCíA MORO, W. F. GADEA AIELLO Y V. DE J. FERNÁNDEZ MORA

\section{Bibliografía}

Altuve, J. G. (20Io). El pensamiento crítico y su inserción en la educación superior. Actualidad Contable Faces, I3(20), 5-I8. Recuperado de https://scielo.conicyt.cl/pdf/estped/v44ni/o7i80705-estped-44-0I-00089.pdf.

Bezanilla Albisna, M. J.; Poblete Ruiz, M.; Fernández Nogueira, D.; Arranz Turnes, S. y Campo Carrasco, L. (20I8). El pensamiento crítico desde la perspectiva de los docentes universitarios. Estudios Pedagógicos, 44(I), 89-II3. doi: http://dx.doi.org/10.4067/ So718-07052018000100089

Bontto, Y.; Vélez, G. y Bono, A. (20ir). Construcción de un Cuestionario de Dilemas para Indagar las Concepciones sobre el Aprendizaje a partir de la Lectura en Ingresantes Universitarios. Summa Psicológica, (8). DOI:I0.18774/448x.20II.8.77.

Boisvert, J. (2004). La formación del pensamiento crítico: Teoría y práctica. México: Fondo de Cultura Económica.

Castaño, C. (1994). Análisis y evaluación de las actitudes de los profesores hacia los medios de enseñanza. Leoia: Servicio de Publicaciones del País Vasco.

Díaz, C.; Ossa, C.; Palma, M.; Lagos, N. y Boudon, J. (2019). El concepto de pensamiento crítico según estudiantes chilenos de pedagogía. Sophia, Colección de Filosofía de la Educación, 26(I), 267-288. doi:https://doi.org/10.17163/soph.n27.2019.09

EnNis, R. (1985). Critical thinking and the curriculum. National Forum, (65), 28-31. Recuperado de https://www.scirp.org/(S(i43dyn45teexjx455qlt3d2q))/reference/ReferencesPapers. aspx?ReferenceID $=278889$.

EnNis, R. (1987). A taxonomy of critical thinking disposition and abilities. En J. E. B. BARON y R. J. Stemberg (eds.), Teaching thinking skills. New York: Freeman. Recuperado de https://www.scirp.org/(S(oyulxb452alntıaejinfow45))/reference/ReferencesPapers.aspx?ReferenceID $=$ I43443I.

Epstein, R. L. (2006). Critical thinking. Belmont, CA: Wadsworth Thomas Learning.

FACIONE, P. (1990). Critical thinking: A statement of expert consensus for purposes of educational assessment and instruction. Millbrae, CA: The Califormia Academic Press.

Febres Cordero, M. A.; Alirio Pérez, A. y Africano Gelves, B. B. (20I7). Las pedagogías alternativas desarrollan el pensamiento crítico. Educere, $2 I(69)$. Recuperado de https:// www.redalyc.org/pdf/356/35649692005.pdf

Franco, A.; Almeida, L. y Saiz, C. (20I4). Pensamiento crítico: reflexión sobre su lugar en la Enseñanza Superior. Educatio Siglo XXI,32(2), 8I-96. doi:http://dox.doi.org/10.6018/j/20217I

García Moro, F. J. (2015). Manual de Competencias Psicológicas del Profesional de la Educación Social. Huelva: Servicios de Publicaciones de la Universidad de Huelva.

García Moro, F. J. (2019). De la crítica del pensamiento al pensamiento crítico. RES, Revista de Educación Social, (29), IIo-II4. Recuperado de http://www.eduso.net/res/winarcdoc. php? id $=1246$.

García Moro, F. J.; Gadea Aiello, W. F. y Chávez, A. (20I9). Modos de pensamiento para el cambio. En prensa.

González, G. (20I2). La educación para la ciudadanía en la formación del profesor de ciencias sociales en Colombia. Representaciones, transiciones y prácticas docentes. Barcelona: Universitat Autónoma de Barcelona.

GonZÁLEZ, G. (20I3). El profesorado en formación y las finalidades de la enseñanza de las ciencias sociales. Revista Unipluriversidad, 38(13), 24-34. Recuperado de https://aprendeenlinea. udea.edu.co/revistas/index.php/unip/article/view/I6971/I4695.

González Valencia, G. y Morillo Puente, S. (2018). Representaciones sobre el desarrollo del pensamiento crítico en maestros en formación. Revista Brasileira de Educação, (23). doi: IO.I590/sI4I3-247820I8230086 
PENSAMIENTO CRÍTICO EN ESTUDIANTES

DEL GRADO DE EDUCACIÓN SOCIAL

F. J. GARCÍA MORO, W. F. GADEA AIELLO Y V. DE J. FERNÁNDEZ MORA

Halpern, D. F. (1998). Teaching critical thinking for transfer across domains. Dispositions, skills, structure training, and metacognitive monitoring. American Psychologist, 53(4), 449455. Recuperado de http://projects.ict.usc.edu/itw/vtt/HalpernAmPsy98CritThink.pdf.

Lipman, M. (1998). Pensamiento complejo y educación. Madrid: De la Torre.

Macchiarola, V. y Martín, E. (2007). Teorías implícitas sobra la planificación educativa. Revista de Educación, 343. Recuperado de http://www.revistaeducacion.educacion.es/re343/ re343_I6.pdf.

Morales ZÚÑIGA, L. C. (20I4). El pensamiento crítico en la teoría educativa contemporánea. Revista Electrónica Actividades Investigativas en Educación, I4(2), I-23. Recuperado de https://www.redalyc.org/pdf/447/44731371022.pdf.

Moreno Pinado, W. E. y Velázquez Tejada, M. E. (2017). Estrategia didáctica para desarrollar el pensamiento crítico. REICE Revista Iberoamericana sobre Calidad, Eficacia y Cambio en Educación, I5(2), 53-73. Recuperado de https://www.redalyc.org/pdf/55I/55150357003. pdf.

Olivares Olivares, S. L. y López Cabrera, M. V. (20I7). Validación de un instrumento para evaluar la autopercepción del pensamiento crítico en estudiantes de medicina. REDIE. Revista Electrónica de Investigación Educativa, I9(2), 67-77. Recuperado de https://www. redalyc.org/pdf/155/15550741006.pdf.

Ossa, C.; Palma Luengo, M.; San Martín, N. y Díaz Larenas, C. (2018). Evaluación del pensamiento crítico y científico en estudiantes de pedagogía de una universidad chilena. Revista Electrónica Educare, 22(2), I-I8. Recuperado de https://www.scielo.sa.cr/pdf/ree/ v22n2/I409-4258-ree-22-02-204.pdf.

Páramo Morales, D. (2015). La teoría fundamentada (Grounded Theory), metodología cualitativa de investigación científica. Pensamiento E Gestión, 39, I-7. Recuperado de https:// www.redalyc.org/pdf/646/64644480oor.pdf.

Paul, R. y Elder, L. (2003). La mini-guía para el pensamiento crítico. Conceptos y herramientas. Dillo Beach: Fundación para el Pensamiento Crítico.

Pithers, R. y Soden, R. (2000). Critical Thinking in Education: A review. Educational Research, 42, 237-249. doi: 10.1080/001318800440579

Reeder, H. P. y Guillén, G. V. (2009). Formación y pensamiento crítico. Revista Interamericana de Investigación, Educación y Pedagogía, 2(I), 23-43. doi:https://doi.org/I0.15332/ si657-107X.2009.0001.02

Rodrigo, M. J. y Correa, N. (1999). Teorías implícitas, modelos mentales y cambio educativo. En J. I. Pozo y C. Moreno (eds.), El aprendizaje estratégico. Enseñar a pensar desde el currículo (pp. 75-85). Madrid: Santillana.

Ruiz, F.; Márquez, C. y Tamayo, Ó. (20I4). Cambio en las concepciones de los docentes sobre la argumentación y su desarrollo en clase de ciencias. Enseñanza de las Ciencias: Revista de Investigación y Experiencias Didácticas, 32(3), 53-70. doi:https://doi.org/I0.5565/ rev/ensciencias.985

Saiz, C. y Nieto, A. M. (2008). Relación entre las habilidades y las disposiciones del pensamiento crítico. Salamanca: Universidad de Salamanca.

SaIz, C. y Rivas, S. (2008). Intervenir para transferir en pensamiento crítico. Praxis, Io(I3), I29I49. Recuperado de http://www.pensamiento-critico.com/archivos/intertranspcpraxis.pdf.

SAIz, C. y Rivas, S. (20I6). New teaching techniques to improve critical thinking. The DIAPROvE methodology. Educational Research Quarterly, 40(o)I, 3-36. Recuperado de http://www. pensamiento-critico.com/archivos/diaproveERQ160307web.pdf

Saiz, C. y Rivas, S. (2017). Pensamiento crítico y cambio. Madrid: Pirámide.

Sánchez, J. L.; Farrán, X. C.; Baiges, E. B. y Suárez-Guerrero, Cr. (20I9). Tratamiento crítico de la información de estudiantes universitarios desde los entornos personales de aprendizaje. Educação e Pesquisa, 45, eI93355. Epub May 30, 2019. doi: https://dx.doi. org/IO.1590/si678-4634201945193355 
F. J. GARCÍA MORO, W. F. GADEA AIELLO Y V. DE J. FERNÁNDEZ MORA

Simon, H. (2oII). El estudio de caso: Teoría y práctica. Madrid: Morata.

Thompson, C. (20II). Critical thinking across the curriculum: Process over output. International Journal of Humanities and Social Science, $I(9)$, I-7. Recuperado de http://www.ijhssnet. com/journals/Vol._I_No._9_Special_Issue_July_2ori/r.pdf.

Valencia, G. y Puente, S. (20i8). Representaciones sobre el desarrollo del pensamiento crítico en maestros en formación. Revista Brasileira de Educação, 23, I-25. doi:http://dx.doi. org/IO.1590/SI4I3-247820I8230086

Valenzuela, J. y Nieto, A. (2008). Motivación y pensamiento crítico: aportes para el estudio de esta relación. Revista Electrónica de Motivación y Emoción, $28(\mathrm{XI})$. Recuperado de http://reme.uji.es/articulos/numero28/article3/article3.pdf.

Valenzuela, J.; Nieto, A. M. y Muñoz, C. (20I4). Motivación y disposiciones: enfoques alternativos para explicar el desempeño de habilidades de pensamiento crítico. Revista Electrónica de Investigación Educativa, I6(3), I6-32. Recuperado de http://redie.uabec.mx/ volı6noz/contenido-valnueto.html.

Velasco Arias, G. (2019). La sociedad del aprendizaje: retos educativos en la sociedad y cultura postmoderna. Revista Prisma Social, 25, 277-298. Recuperado de https://revistaprismasocial.es/article/view/2735.

Vélez Gutiérrez, C. F. (20I3). Una reflexión interdisciplinar sobre el pensamiento crítico. Revista Latinoamericana de Estudios Educativos, 9(2), II-39. Recuperado de http://vip.ucaldas.edu.co/latinoamericana/downloads/Latinoamericana9(2)_2.pdf

Wolton, D. (20I0). Informar no es comunicar. Barcelona: Gedisa. 
